\title{
Manual therapy program induced alterations in autonomic nervous system modulation of students preparing to apply for the universities: One-group, pre- and post-test study
}

\author{
Heloísa Aparecida, Filipy Borghi, Gustavo Trevisan Costa, Fernanda Vasconcellos Bortz, Priscila Cristina da Silva, Dora Maria Grassi-Kassisse*
}

Ferreira HA, Borghi F, Costa GT, et al. Manual therapy program induced alterations in autonomic nervous system modulation of students preparing to apply for the universities: One-group, pre-, and post-test study. Curr Integr Res Med 2017;2(2):21-25.

OBJECTIVES: We aimed to appraise the effects of a manual therapy program (MTP) on stress by modulation of autonomic nervous system evaluated by rate heart variability before and after the treatment.

METHODS: The study design used was quasi-experimental, pre-post-test of MTP. The sessions were applied during $45 \mathrm{~min}$, twice a week, for 9 weeks. Psychological stress was evaluated using adolescents stress questionnaire (ASQ). Beat-to-beat rate heart frequency (HR, bpm) were recorded in 16 young healthy volunteers (19-20 years old; 8 women) 15 min before and after the first and the last session with S810 i - Polar ${ }^{\circledR}$-Finland in supine position. Descriptors of the Poincaré plot and time domain of HR variability (HRV) were analyzed in 5 min (Kubios HRV Analysis Software).

STATISTICAL ANALYSIS: ANOVA followed Tukey or Bonferroni and $t$ Student tests paired and unpaired tests, considering $\mathrm{p}<0.05$ as significant.

RESULTS: MTP reduced ASQ values from $143 \pm 8$ to $114 \pm 6$ and HR from $81+4$ to $71+2 \mathrm{bpm}$. Significantly effects were also found post-MTP in HRV time domain, analyzed at linear and geometric way, sowing an overall increased on parasympathetic tonus and on global activity of heart.

CONCLUSION: The students enrolled in the MTP showed improved psychological and physiological health status from pre to post-treatment and the increased on parasympathetic tonus and in the global activity of heart contributed to this effect.

Key Words: Adolescents; Manual massage; Heart rate variability; Sympathetic nervous system; Parasympathetic nervous system
$T^{1}$

he application to join Brazilian universities is hard and an agent that increases stress reaction in adolescents. The main problem is the low vacancy number at public universities, i.e. higher competition. In one state university, which offers 3.320 vacancies, received an increased number of inscriptions: 67.516 in 2014; 77.146 in 2015 and 77.760 in 2016, enhance of almost $16 \%$ in three years (1). The federal system is not different, in 2013 received more than 2.712 .937 inscriptions to 171,401 vacancies of different universities (2).

However, the competition test is only an additional stress agent for students in this age. Of all life-stages adolescence is arguably the one most marked by rapid and potentially tumultuous transition, including biological, social, and psychological changes as well as shifting self-concepts (3).

The association between stress, self-esteem and emotional outcomes as well as gender differences on these constructs is helpful investigation for health promotion as preventive strategies during adolescence. Research has established that overall levels of stress tend to increase from preadolescence to adolescence. Evidence suggests that adolescent exposure to multiple independent and cumulative stressors, especially those in an interpersonal context, is related to psychological symptomatology of clinical significance, including symptoms of depression and anxiety. Studies also provide evidence that stressful life experiences predict increases in psychological problems over time (4).

A growing number of studies with adolescents and stress reactions and feelings are available in the literature. According to the World Health Organization, stress is a significant problem of our life time and affects physical and mental health. Stress is defined as a state of threatened or perceived as threatened homeostasis (5) and stress coping methods are the cognitive, behavioral and psychological efforts to deal with stress (6-8).

Stress managements are well studied in adults. Varvogli and Darviri (8) realize a review in major databases (MEDLINE, Scopus, Science Direct) with different relaxation techniques, for example: biofeedback, diaphragmatic breathing, and transcendental meditation. These authors conclude that these techniques are all evidence-based, easy to learn and practice and present positive results in individuals with good health or with a disease.
In another study, a stress management program based on cognitive behavioral therapy principles was compared with a Kundalini yoga program. The results indicate that both cognitive behavior therapy and yoga are promising stress management techniques (9). Batista et al. related the positive effect of tantric yoga program as eustress promoter (10).

Therefore, we aimed to evaluate the effects of manual therapy program as stress coping in adolescents who are preparing to apply for universities, analyzing cardiac modulation of autonomic nervous system and adolescent stress questionnaire.

\section{METHODS}

The protocol conduced was one-group pre and post-test design (11). The participants enrolled in the manual therapy program (MTP) and completed self-report questionnaire of perceived stress for adolescents at pre-treatment and after 9 weeks post-treatment. We only considered for this study the volunteers which have participated in all MTP and collected all samples $(n=16)$. The protocol was approved by the Ethical Committee of Medical Science Faculty of Universidade Estadual de Campinas as addendum (no.27/10/2009) of the main Project n³88/2006-FR102878 (CONEP).

\section{Participants and procedure}

The primary outcome measure was to be student at preparatory course to apply for universities. This study was performed with collaboration of students from "Curso Preparatório para o Vestibular Cooperativa do Saber", located at Street 14 de Dezembro, Campinas, São Paulo, Brazil. There were many lectures to clarify the study for the school, parents and students. A heterogeneous group with women (W, $n=8)$ and men $(M, n=8)$ has formed.

Participants met the following inclusion criteria: 1- Be a student attending prep-school and pre-college, enrolled at school at morning classes, and aged between 16 and 20 years; 2- Present yourself as a volunteer after receiving information about this study, sign the informed consent form and a health declaration; 3- Have parental authorization (in the case of patients younger than 18 years). Exclusion criteria were: 1-Make use of steroid hormones and/ or cigarettes; 2- Present a chronic disease; 3- Suffered some causative factor 
of stress, such as death of close people, recent divorce of parents, moving to a new house, among others, in the last six months; 4- Pregnancy in the case of female volunteers.

\section{Intervention - manual therapy program}

Volunteers were treated at school, in a room specially prepared for the procedures. Volunteers remained at rest for $10 \mathrm{~min}$ in the supine position before starting the procedure. The massage involved entire student body and was started in supine position. Manual therapy (MT) consisted of movements in the all muscle fascias even in the face. MT was done for 45 min, twice a week, for nine weeks, starting in September and finishing in November. Every session of the study took place at the same time of day. MT was performed by six women physiotherapists specially trained, and did not play another role in the study. There were no conversations during the study, only a few times answering participant's questions, before or after procedure. This procedure was done to be sure that the effects obtained came from MT program $(12,13)$. Heart rate registrations were carried out before and at the end of the first and the last intervention.

\section{Measurements and analyses}

All participants completed a composite questionnaire assessing:

a) Basic information (age, sex, weight, height, chronic disease, familiar diseases, if use some chronic medicaments);

b) Psychological stress evaluation - Adolescent Stress Questionnaire (ASQ) (3).

Questionnaire administration was conducted in whole class groups at school, before starts the procedure and after the last session of manual therapy. Volunteers were free to fill it out individually and without interference from others, and to the parental authorization if the volunteers are younger than 18 years. The questionnaires were tabulated for use with paired data discarding unpaired data. This questionnaire was adapted to Portuguese Language by Prof Bratfisch under Prof Byrne supervision and validated by Ferreira (14). It is a 62-item inventories related to common adolescent stressors. Each item (stressor) was rated on a 5-point scale where 1 not at all stressful (or is irrelevant to me); 2 a little stressful; 3 moderately stressful; 4 quite stressful; and 5 very stressful. The students were oriented to answers the ASQ about stressor exposure in the last six months from September, and at the end of the MTP, considering the events which happened in the last two months of the program.

Considering the relevance of social relationships in Brazilian students' profile, it was included additional four questions and a new scale-stress of social relations with authors approve. For the purposes of deriving selfreported adolescent stress, scores reflecting the extent to which individual respondents were characterized by each of the remaining 11 components (scales) of adolescent stressor experience were calculated simply by summing the affirmed response to each item (1 to 5) across all items defining the component of each 62 questions (13).

\section{Heart rate and heart rate variability}

The activity in the autonomic nervous system (both sympathetic and parasympathetic) was estimated based on analysis of heart rate variability (HRV), which refers to the beat-to-beat fluctuations in heart rate (HR). Beatto-beat rate heart frequency $(\mathrm{HR}, \mathrm{bpm})$ were recorded in 16 young healthy volunteers $15 \mathrm{~min}$ before and after the first and the last session with S810i - Polar ${ }^{\circledR}$-Finland in supine position. Data were stored on a computer for further analysis using custom made software. There were no records during massage period. Descriptors of the statistical and geometrical time domain Poincaré plot, and frequency domain of HRV were analyzed for each interpolated 5 min central recorded (Kubios HRV Analysis Software).

\section{Statistical analysis}

All data are reported as mean \pm SEM, and differences were considered significant when $\mathrm{p}<0.05$. After normality test performed by Shapiro-Wilk according to Graph Pad Prisma (Version 9.2; SAS Institute Inc, 2002-2008, Cary, NC, USA) suggestion, the tests utilized were Wilcoxon or Student t test paired for non-parametric or parametric when suitable, respectively.

\section{RESULTS}

\section{Volunteer characterizations}

Volunteers of this study were aged between 18 and 20 years old and all of them were eutrophic (BMI, 22.5+0.8). There were no differences between men $(\mathrm{M})$ and women (W) concerning about age, but there were differences in BMI data $(W=20.9+1.1$ vs. $M=24.0+0.9$, $p<0.05)$.

Psychological stress evaluation - Adolescent Stress Questionnaire (ASQ)

MTP reduced ASQ values from $143 \pm 8$ to $114 \pm 6, p=0.0005$. When we consider the sex, there were also a reduction, and for this index, there were no difference between women $(145+12$ vs. $126+8)$ and men $(141+13$ vs. $103+7)$

\section{Heart rate $(\mathrm{HR})$}

There was a reduction of HR after the first and the last session of MT. Besides, it is interesting to note that the HR values recorded at the time before the last session were lower than those at the beginning of the treatment. Analysis of the results considering sex showed that women and men present similar responses after MTP, although the reduction had been significant only after the last session of MT (Figure 1).

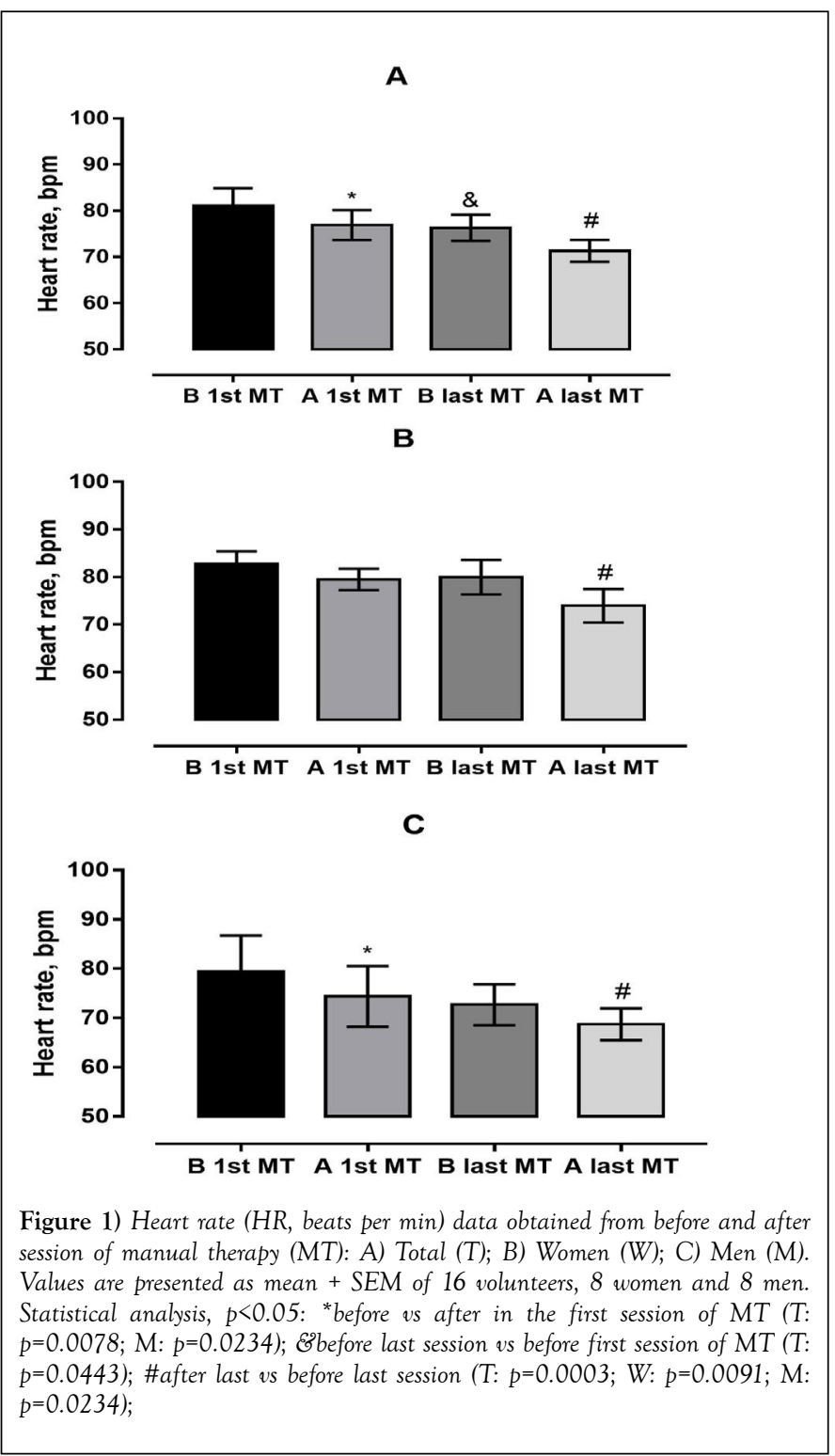

Heart rate variability

Time domain: Statistical methods

The analysis of statistical indicators of heart rate variability-time domain (RMSSD, ms and pNN50 \%) showed an increase in parasympathetic modulation of both after the first and the last session of MT, in total population and in women (Figure 2). 


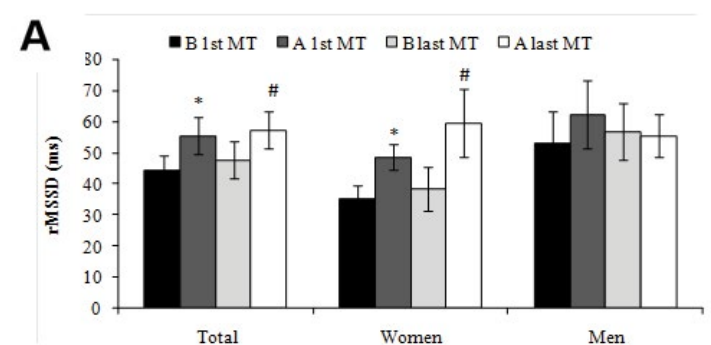

B

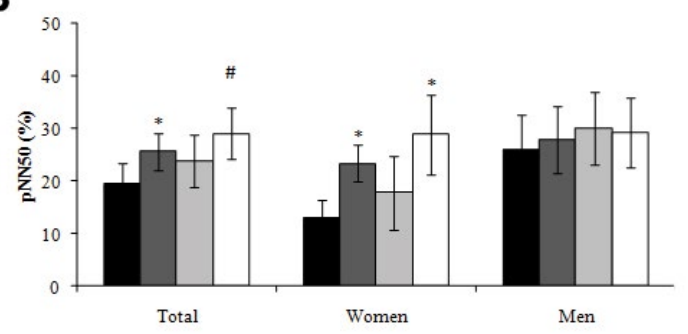

Figure 2) Statistical index of time domain obtained from iRR' adjacents in 5 min. Data $r$ MSSD (ms, A) and pNN50 (\%, B) before and after the first and the last session of manual therapy (MT). Values are presented as mean + SEM of 16 volunteers, 8 women and 8 men. Statistical analysis, $p<0.05 ; r$ MSSD: *before vs after in the first session of MT (T: $p=0.0084 ; \mathrm{W}: p=0.0158$ ); \#after last vs before last session (T: $p=0.0257 ; \mathrm{W}: p=0.0056$ ). $p$ NN50: *before ws. after in the first session of MT (T: $p=0.0151 ; W: p=0.0077)$; \#after last us. before last session (T: $p=0.0412 ; W: p=0.0089$ ).

\section{Time domain: Geometric methods}

Figure 3 illustrates typical records obtained from a woman and a man before the first and after the last session of MT. The qualitative analysis (visual) of the Poincaré plots shows the obvious effect of MT in causing greater dispersion of RR intervals beat by beat. It is possible to observe an increase in the dispersion of both points along the line of identity (SD2) and perpendicular to the line (SD1). Quantitative analysis (Figure 4) evaluating the average of SD1, SD2 and SD1/SD2 demonstrate an increase in parasympathetic tone (SD1) activity in both the total population and women indexes. These results also show an increase in the overall activity of the heart (SD2) of all volunteers and women, and after the first session in male. This indicator (SD2) also shows differences in baselines (before first and last sessions) between men and women, being higher in men. The reason SD1/ $\mathrm{SD} 2$ remained unchanged throughout the program.
A

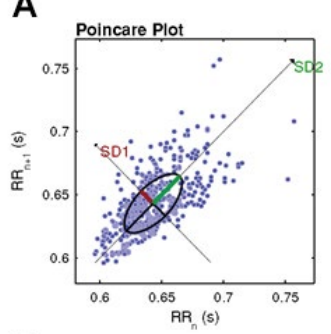

C

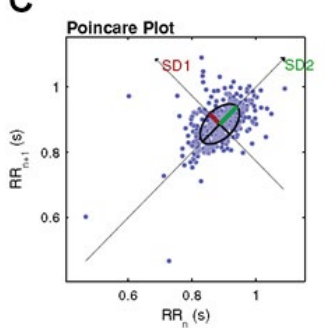

B

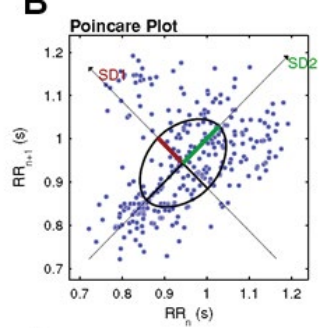

D

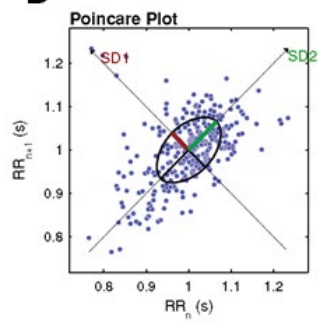

Figure 3) Point-caré plots showing geometrical qualitative visual analysis of time domain of two volunteers. A: women before the first MT session; B: women after the last MT session; C: men before the first MT session and D: men after the last MT session. Each R-R interval $(R R n+1)$ is plotted as a function of previous RRn interval
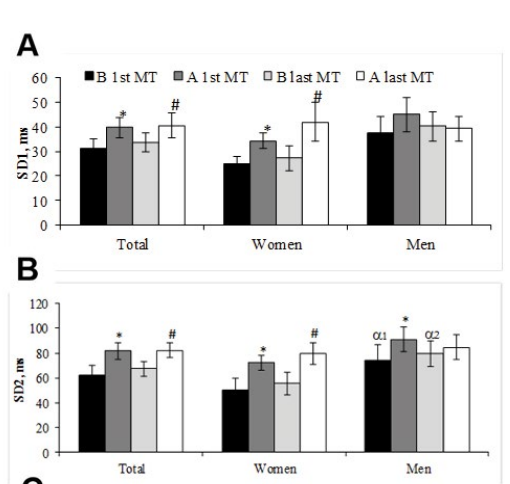

C

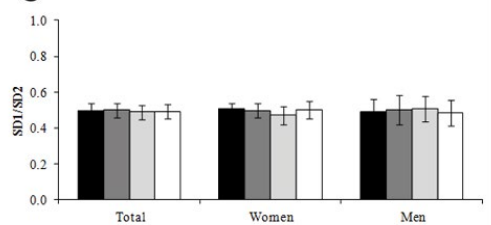

Figure 4) Geometrical quantitative analysis of time domain. A: SD1 - the standard deviation measuring the dispersion of points in the plot across the identity line. This parameter is usually interpreted as a measure of short-term HRV. B SD2 - The standard deviation measuring the dispersion of points along the identity line and C: SD1/SD2 ratio. All points are projected on the identity line, and the standard deviation is calculated. This variable is interpreted as a measure of both short- and long-term HRV. Data were collected before and after the first and the last session of manual therapy (MT). Values are presented as mean + SEM of 16 volunteers, 8 women and 8 men. Statistical analysis, $p<0.05$ : SD1: *before vs. after in the first session of $\mathrm{MT}(\mathrm{T}: \mathrm{p}=0.0053$; W: $p=0.0158)$; \#after last vs. before last session ( $T: p=0.0255 ; \mathrm{W}: p=0.0055) ; \mathrm{SD2}$ : * before vs. after in the first session of $\mathrm{MT}(\mathrm{T}: \mathrm{p}=0.0043 ; \mathrm{W}: \mathrm{p}=0.0425, \mathrm{M}: \mathrm{p}=0.0281)$; \#after last vs before last session ( $T: p=0.0067 ; W: p=0.0015) ; \alpha$ lbefore first men ws before first women $(p=0.0489) ; \alpha 2$ before last men vs before last women $(p=0.0305)$.

\section{Frequency domain}

At the beginning of assay, the data collected were: $\mathrm{LH}$ : 46.3+4.3; HF $53.7+4.3$ and ratio $L F / H F 1.11+0.2$. These data did not change after manual therapy (Figure 5).

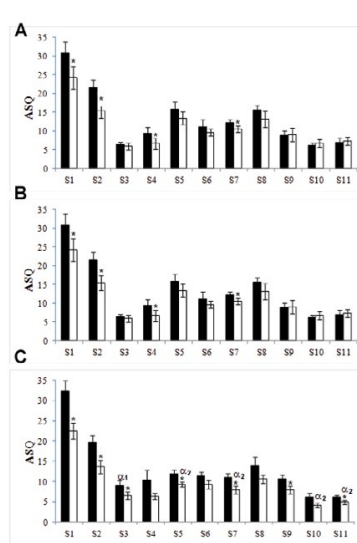

Figure 5) Adolescent Stress Questionnaire (ASQ) scores: A: total; B: women; C: men; volunteers obtained before and after MTP. ASQ is composed by 11 different stressors of adolescent life. S1-Stress of home life; S2-Stress of school performance; S3-Stress of school attendance; S4-Stress of romantic relationships; S5-Stress of peer pressure; S6-Stress of teacher interaction; S7-Stress of future uncertainty; S8-Stress of school/Leisure conflict; S9-Stress of financial pressure; S10-Stress of emerging adult responsibility; S11-Stress of social relationship. Data were collected before and after nine weeks of manual therapy program. Values are presented as mean + SEM of 16 volunteers, 8 women and 8 men. Statistical analysis, $p<0.05$ : *after vs. before (T: S1, $p=0.0003 ; \mathrm{S} 2, p=0.0005 ; \mathrm{S} 4, p=0.0137 ; \mathrm{S} 7, p=0.0023 ; \mathrm{W}$ : $S 1, p=0.0388 ; S 2, p=0.0226 ; S 4, p=0.0450 ; S 7, p=0.0241 ; M: S 1, p=0.0008$; S2, $p=0.0060 ; S 3, p=0.0021 ; S 5, p=0.0025$; S7, $p=0.0202 ; S 9, p=0.0462$, $\mathrm{S} 11, p=0.0192) \alpha 1$ before men vs. before women (S3: $p=0.0410$ ); $\alpha 2$ After men vs. after women (S5: $p=0.0181 ; \mathrm{S} 7: p=0.0478 ;$ S10, $p=0.0141, \mathrm{~S} 11, p=0.0367$ ). 


\section{DISCUSSION}

The proposed program of manual therapy was effective in improving stress indexes assessed by stress questionnaire for adolescents. The ASQ was developed to address the requirement for systematic research examining adolescent stress in the early 21st century. Byrne and colleagues state that the ASQ is not a measure of symptomatic distress although it assesses the subjective stressor load. The items are statements concerning events or situations which adolescents could find stressful. The ASQ has a good construct validity (positive correlations with measures of anxiety and depression, and negative correlations with self-esteem), internal and testretest reliability, and demonstrates consistent gender differences (girls report higher stress levels than boys), but poor correlations with age for certain stress dimensions $(14,15)$. The items were generated in adolescent focus groups, where they brainstormed on the concerns and challenges associated with adolescence that had affected them or their peers, and for Brazilian students were included one scale with questions about social relationship (13). The items were attributed to 11 scales or dimensions of adolescent stressor experiences (S1- stress of home life, S2- school performance, S3school attendance, S4- romantic relationships, S5- peer pressure, S6- teacher interaction, S7- future uncertainty, S8- school/leisure conflict, S9- financial pressure, S10- emerging adult responsibility and S11- social relationship). These component scales were thematically meaningful within the existing body of theory and knowledge regarding the experience of adolescent stress $(3,13,15)$.

The items with higher score of our study are coincident with Byrne's colleague studies, however, in their study showed differences, with girls higher than boys in S1, S2, S4, S5, S7, S8, S10 scales. In our study, we find differences before starting the program only at school attendance (S3). The main difference between the studies is about the age, while Byrne (3) has 15 years old volunteers, ours students are 19 years old and are under one additional stressful agent, which is to prepare themselves for apply to universities. MT begot reduction in scores related to stress of home life, school performance, school attendance, romantic relationships, peer pressure, future uncertainty and school/leisure conflict. The worries about teacher interaction, financial pressure, emerging adult responsibility and stress social relationship remains the same as showed before MT starts.

ASQ scores were also evaluated before and after MT considering the sex, where women and men responded differently: both showed reduction on home life (S1), school performance (S2), and future uncertainty (S7) scales. Women presented also reduction in romantic relationships (S4) scores and men showed additional reduction in school attendance (S3), peer pressure (S5), financial pressure (S9), and social relationship (S11) scores.

However, some items, even with low scores, remain stressful for Brazilian students. Women kept worried about school attendance, peer pressure, teacher interaction, school/leisure conflict, financial pressure, emerging adult responsibility and stress social relationship and men with romantic relationships, teacher interaction, school/leisure conflict and emerging adult responsibility. These results suggest that massage therapy program was able to improve stress coping in students.

Indeed studies conducted in populations with different age groups indicate the efficiency of different relaxation techniques in decrease distress levels (16). Massage therapy, one of the oldest treatment modalities, has been reemerging as one of the most popular forms of alternative therapy. Despite its long history, controlled studies have only recently been conducted to assess the biochemical, physiological, cognitive, and emotional effects of massage therapy $(17,18)$.

MT also induced in students a decrease in heart rate after the first session and the effect was maintained even after nine weeks. We also observed that the basal HR, ie, the time before the last session was reduced, suggesting that frequency of this practice may induce cardiac protection.

In fact a meta-analysis of massage therapy research showed that a single application of MT reduced state anxiety, blood pressure, and heart rate but not negative mood, immediate assessment of pain, and cortisol level in adults (19).

It is already described that MT may provide its benefits by shifting the autonomic nervous system (ANS) from a state of sympathetic response to a state of parasympathetic response and is associated with decreased cardiovascular activity, a decrease in stress hormones, and feelings of calmness and wellbeing (20). Some researches shows that the pressure applied during MT may stimulate vagal activity (18), which in turn leads to a reduction of stress hormones and physiological arousal, and a subsequent parasympathetic response of the ANS (19). Through physiological parasympathetic stimulating, MT may promote reductions in anxiety, depression, and pain that are consistent with a state of calmness. This same mechanism may also be responsible for several condition-specific benefits resulting from MT, such as increased immune system response in HIV-positive individuals (21), or improved mental performance function during a test with changes in electroencephalography pattern consistent with increased relaxation and alertness (22). This theory is not supported universally, and it has even been suggested a long time ago that MT may promote a sympathetic response of the ANS (23).

Researchers evaluates the physiological and psychological effects of three different massage therapy techniques, including light pressure and deep pressure massage provided by hands and vibratory stimulation provided by a mechanical massager. This study shows that moderate changes in anxiety and stress were assessed. Anxiety scores decreased for all groups, but the moderate pressure massage group reported the greatest decrease in stress. The moderate massage group also experienced a decrease in heart rate and EEG changes including an increase in delta and a decrease in alpha and beta activity, suggesting a relaxation response at this group (24).

However, as our MT was considered as moderate we suggested that the decrease in HR values may be due to an increase in the parasympathetic nervous system activity instead of a reduction of sympathetic activity on the heart (25). To clarify this effect, we evaluate the heart rate variability.

Studies of heart rate variability are becoming more frequent as they are not invasive and inexpensive measures. However, due to the range of information arising from this analysis in 1996, a task force with researchers from many places in the world to establish criteria and indicators were performed. Heart rate variability (HRV), the variation over time of the period between consecutive heartbeats, is predominantly dependent on the extrinsic regulation of the heart rate (HR). The degree of variability in the HR provides information about the functioning of the nervous control on the HR and the heart's ability to respond $(25,26)$.

Many HRV measures have been proposed, which can roughly be subdivided into time domain, frequency domain and nonlinear domain measures. The most commonly used measures derived from interval differences include RMSSD (the square root of the mean squared differences of successive NN intervals), NN50 (the number of interval differences of successive NN intervals greater than $50 \mathrm{~ms}$ ) and pNN50 (the proportion derived by dividing NN50 by the total number of NN intervals). Two types of HRV indices are distinguished in time domain analysis. Both types of indices are calculated from the RR intervals occurring in a chosen time window (usually between 0.5 and $5 \mathrm{~min}$ ). The statistical parameters SDNN, SENN, SDSD, RMSSD, NN50 (\%), and pNN50\% can be used as time domain parameters $(25,26)$.

SDNN, and SDANN SDNNi indices are derived from long-term records and represent the sympathetic and parasympathetic activity, but cannot distinguish when changes in HRV are due to increased sympathetic tone or by withdrawal of vagal tone. The RMSSD and pNN50 represent parasympathetic activity; they are calculated from the analysis of adjacent RR intervals (26-28).

Among the methods used for HRV analysis, we may include the Poincaré plot, a dimensional graphical representation of the correlation between consecutive RR intervals, in which each RR interval is plotted against the next interval. The plot Poincaré may be quantitatively analyzed by means of the following indexes: SD1, SD2 and SD1/SD2 obtained by fitting the ellipse formed by the attractor of the figure. It may be qualitatively assessed through the shape formed by its attractor, which shows the degree of RR intervals complexity. The the Poincaré plot analysis has been considered by some authors as a nonlinear dynamics method. Although it is still scarce in the clinical literature, the nonlinear methods helps to understand the system behavior that linear models do not explain, since there is evidence that the mechanisms involved in cardiovascular regulation likely interact in a nonlinear way (29).

Tulppo et al. (30) described that quantitative two-dimensional vector analysis of a Poincaré plot (SD1, SD2 and SD1/SD2) can provide useful information on vagal modulation of R-R interval dynamics during exercise that are not easily detected by linear summary measures of HRV. Consequently, in our studies, the variability of HR was evaluated in time domain analysis (RMSSD and pNN50), nonlinear geometric measures by Poincaré plot (SD1, SD2 and $\mathrm{SD} 1 / \mathrm{SD} 2)$.

The Poincaré plot recorded in our study demonstrate shape and basal data of RMSSD, SD1, SD2 ratio SD1/SD2 and LF, HF and ratio LF/ 
HF quite similar to Vanderlei studies for participants aged between 17-18 years old which evaluate the heart rate variability in healthy adolescents at rest (29). There were significantly effects of manual therapy after a single session and also after all nine weeks of treatment related to an increase on parasympathetic tonus of autonomic system.

In 2016, Field presented a review in literature highlighting that the massage therapy groups have experienced more positive effects than the control or comparison groups. This may relate to the massage therapy providing more stimulation of pressure receptors, in turn enhancing vagal activity and reducing cortisol levels. Some of the researchers have assessed physical, physiological and biochemical effects, although most have relied exclusively on self-report measures. Despite these methodological problems and the dearth of research from the U.S., the massage therapy profession has grown significantly and massage therapy is increasingly practiced in traditional medical settings, highlighting the need for more rigorous research (31).

\section{CONCLUSION}

We can conclude that MT program suggested reduced adolescent stress evaluated by ASQ and HR values, indicating efficiency of this therapy on managing distress. These effects were attributed to enlargements of the parasympathetic tonus and the global activity evaluated by HRV time domain. We can point out that MT's benefits are evident over the population which had the parasympathetic tonus and the global activity lower.

\section{Study limitations}

When evaluating massage, it is impossible to blind the treatment. There is always an interpersonal component that can affect the treatment. The concept of touch massage involves physical, emotional, cognitive, social and interpersonal aspects.

\section{ACKNOWLEDGEMENT}

We would like to thank the students who participated in this study. We thank the University of Campinas (UNICAMP), the Fund for Research, Teaching and Extension Support (FAEPEX), the Research Support Fund (FAP) of University Metodista de Piracicaba (UNIMEP) and São Paulo Research Foundation (FAPESP - 06/532664) for the financial support. Capes for scholarship. We also thank SH and GS for the language services provided. The study is part of a PhD Program done by Heloísa Aparecida Ferreira and supervised by Prof Dr Dora Maria Grassi-Kassisse.

\section{REFERENCES}

1. Vestibulares Anteriores - COMVEST 2017 [Available from: http://www. comvest.unicamp.br/vestibulares-anteriores/].

2. Sisu - Sistema de Seleção Unificada 2017 [Available from: http://sisu. mec.gov.br/].

3. Byrne DG, Davenport SC, Mazanov J. Profiles of adolescent stress: the development of the adolescent stress questionnaire (ASQ). J Adolesc. 2007;30(3):393-416

4. Moksnes UK. Faculty of Nursing STUC, Trondheim, Norway, Research Centre for Health Promotion and Resources HiST/NTNU T, Norway, Department of Social Work and Health Science NUoSaT, NTNU, 7491 Trondheim, Norway, Rannestad T, Faculty of Nursing STUC, Trondheim, Norway. The association between stress, sense of coherence and subjective health complaints in adolescents: Sense of coherence as a potential moderator. Stress and Health. 2017;27(3).

5. Charmandari E, Tsigos C, Chrousos G. Endocrinology of the stress response. Annu Rev Physiol. 2005;67:259-84.

6. Arunabh S, Pollack S, Yeh J, et al. Body fat content and 25-hydroxyvitamin D levels in healthy women. J Clin Endocrinol Metab. 2003;88(1):157-61.

7. Baumann A, Muijen M. Mental health and well-being at the workplace - protection and inclusion in challenging times (2010): World Health Organization; 2010.

8. Varvogli L, Darviri C. Stress management techniques: evidence-based procedures that reduce stress and promote health. Health Science Journal. 2011;5(2).

9. Granath J, Ingvarsson S, Von Thiele U, et al. Stress management: a randomized study of cognitive behavioural therapy and yoga. Cogn Behav Ther. 2006;35(1):3-10.
10. Batista JC, Souza AL, Ferreira HA, et al. Acute and chronic effects of tantric yoga practice on distress index. J Altern Complement Med. 2015;21(11):681-5.

11. Vriezekolk JE, Eijsbouts AM, Van Lankveld WG, et al. An acceptanceoriented cognitive-behavioral therapy in multimodal rehabilitation: A pre-post test evaluation in highly distressed patients with rheumatic diseases. Patient Educ Couns. 2013;91(3):357-63.

12. Lindgren L, Rundgren S, Winso O, et al. Physiological responses to touch massage in healthy volunteers. Auton Neurosci. 2010;158(1-2):105-10.

13. Ferreira HA. Effect of a manual therapy program (MTP) on heart rate variability (HRV) and on indicators of stress in school students. SBU: University of Campinas; 2014.

14.Ferreira, AH. Manual physiotherapy intervention reduces stress levels in students during exam period. SBU: Universidade Estadual de Campinas; 2009.

15. De Vriendt T, Clays E, Moreno LA, et al. Reliability and validity of the adolescent stress questionnaire in a sample of European adolescents-the HELENA study. BMC Public Health. 2011;11:717.

16. Van der Klink JJ, Blonk RW, Schene AH, et al. The benefits of interventions for work-related stress. Am J Public Health. 2001;91(2):270-6.

17. Field T, Schanberg S, Kuhn C, et al. Bulimic adolescent's benefit from massage therapy. Adolescence. 1998;33(131):555-63.

18. Field T, Hernandez-Reif M, Diego M, et al. Cortisol decreases and serotonin and dopamine increase following massage therapy. Int J Neurosci. 2005;115(10):1397-413

19. Moyer CA, Rounds J, Hannum JW. A meta-analysis of massage therapy research. Psychological Bulletin. 2004;130(1):3.

20.Sarafino EP, Smith TW. Health psychology: Biopsychosocial Interactions, 8th edition: Wiley; 2014 January 2014, Â@2014.

21.Diego MA, Field T, Hernandez-Reif M, et al. HIV adolescents show improved immune function following massage therapy. Int J Neurosci. 2001;106(1-2):35-45.

22. Field T, Ironson G, Scafidi F, et al. Massage therapy reduces anxiety and enhances EEG pattern of alertness and math computations. Int J Neurosci. 1996;86(3-4):197-205.

23. Barr JS, Taslitz $\mathrm{N}$. The influence of back massage on autonomic functions. Phys Ther. 1970;50(12):1679-91.

24.Diego MA, Field T, Sanders C, et al. Massage therapy of moderate and light pressure and vibrator effects on EEG and heart rate. Int J Neurosci. 2004;114(1):31-44.

25. Rajendra Acharya U, Paul Joseph K, Kannathal N, et al. Heart rate variability: a review. Med Biol Eng Comput. 2006;44(12):1031-51.

26. North American Society of Pacing and Electrophysiology. Heart rate variability: Standards of measurement, physiological interpretation and clinical use. Task Force of the European Society of Cardiology. 93 Circulation, 1996.

27.Pumprla J, Howorka K, Groves D, et al. Functional assessment of heart rate variability: physiological basis and practical applications. Int J Cardiol. 2002;84(1):1-14

28. Vanderlei LC, Pastre CM, Hoshi RA, et al. Basic notions of heart rate variability and its clinical applicability. Rev Bras Cir Cardiovasc. 2009;24(2):205-17.

29.Vanderlei FM, Rossi RC, Souza NM, et al. Heart rate variability in healthy adolescents at rest. Journal of Human Growth and Development. 2012;22(2):173-8.

30.Tulppo MP, Makikallio TH, Takala TE, et al. Quantitative beat-to-beat analysis of heart rate dynamics during exercise. Am J Physiol. 1996;271(1 Pt 2):H244-52.

31. Field T. Massage therapy research review. Complement Ther Clin Pract. 2016;24:19-31 